\title{
Loving Touch: Treatment for Rejection of Care Ladislav Volicer*
}

School of Aging Studies, University of South Florida, USA

*Corresponding author: Ladislav Volicer, School of Aging Studies, University of South Florida, Tampa, 2337 Dekan Lane Land O Lakes, FL 34639, USA, Tel: + (813) 909-0539; E-mail: Ivolicer@usf.edu

Received date: December 14, 2015; Accepted date: December 19, 2015; Published date: December 26, 2015

Copyright: @ 2015 Volicer L, et al. This is an open-access article distributed under the terms of the Creative Commons Attribution License, which permits unrestricted use, distribution, and reproduction in any medium, provided the original author and source are credited.

\section{Editorial}

Alzheimer's disease is a major problem for the health care system because its prevalence is increasing with an aging population. There is no effective medication, which would prevent or treat Alzheimer's disease on the horizon, and therefore appropriate care for people with this disease is increasingly important. In addition to cognitive deficits that result in functional impairments and a need for care partner, people with dementia often exhibit behaviors that may be more disturbing than the cognitive deficits. Care partners are most disturbed if people with dementia do not cooperate with their caregiving efforts and reject care [1]. People with dementia may actually fight with care partners if they feel that the care is not necessary, or if they do not understand the care partners' intentions.

Often, such people are labeled as aggressive or assaultive. This label is actually blaming the victim, because people with dementia may consider the care partner, who insists on providing care, as the aggressor. If rejection of care occurs in a home situation, the result may be institutionalization of the person with dementia [2]. If rejection of care occurs in an institutional setting, the result is often the administration of a psychoactive medication, e.g., an antipsychotic [3]. Antipsychotics have many adverse effects, including increased risk of stroke and sudden death. In addition, combative behavior that can develop when the person with dementia rejects care may result in an injury of the person with dementia or the care partner. Therefore, it is important to provide care partners with approaches they can use if the person with dementia rejects their care, which does not include administration of a medication.

Conditions related to rejection of care include intrinsic factors (health state and psychosocial factors), antecedents (personal care processes, physical environment, and social interaction), and match between needs and environmental resources [1]. However, the two most important factors are inability to communicate and depression [4]. Several studies were performed that tried to influence one or more of these factors. The most effective were strategies using aromatherapy, ability-focused care partner education, bed bath, preferred music, and muscle relaxation training. However, only some participants benefited from these interventions [5]. Often, nursing home staff is not aware of these strategies or does not apply them because they are not part of their usual practice. This results in a battle between residents and staff, if the rejection of care escalates into combative behavior.

This battle may not be seen by others because it occurs most often in residents' rooms, but it is detrimental for the staff and residents. In addition to possible injuries, it leads to frustration, nervousness, fear, and distress in nursing assistants and decreased quality of life for residents. Nursing aides in Canada reported that residents defend themselves by slapping, squeezing, punching, hitting, and shoving the nursing home staff and these behaviors indicate a high level of stress the residents perceive. What is most discouraging is that most of these nursing aids believed that they cannot control, change, or modify this situation. Most of them continued the battle to provide care [6]. Therefore, there is a need for program that would institutionalize a strategy that would become standard of care. One of such program is Namaste Care.

Namaste Care is a program that was developed for people with dementia who can no longer engage in traditional activity programs [7]. The two main principles of this program are special environment and loving touch. Namaste care takes place in a calm environment that is as free of distractions as possible, where a small group of residents meets four hours a day seven days a week. The loving touch approach is provided as a hand or foot massage, application of a familiar scented lotion to the face, and massage of the scalp. This program resulted in improved quality of life [8] and decreased behavioral symptoms of dementia [9]. It also led to the discontinuation of antipsychotic and hypnotic medications [10]. A qualitative study indicated that the loving touch approach led to more engagement of residents with the environment and each other, better communication between the residents and both staff and family members. The study also found decreased pain in residents, and decreased rejection of care.

Loving touch approach decreases rejection of care because residents are exposed to loving touch repeatedly during Namaste Care session. This experience makes them more tactile; more accepting being touched and this tolerance transfers also to touch necessary for provision of care. Staff reported that "Challenging behavior has reduced, is now cooperative in helping to get dressed" and "He is less resistant to care delivered by female carers, easier to care for, does not complain of pain during care" [8]. The staff found their job less challenging and more satisfying after implementation of Namaste Care. Namaste Care does not require additional staff, expensive equipment or supplies. The environment and loving touch approaches can be offered in skilled nursing facilities, assisted living communities, and in a persons' home. One nationwide hospice organization offers Namaste Care to their patients with advanced dementia.

Beneficial effects of loving touch are depicted in Figure 1. Loving touch prevents rejection of care by increasing tolerance for touch, improving communication between residents and care partners and decreasing depression [11]. Decreased or eliminated rejection of care prevents combative behavior that is stressful and dangerous for both residents and staff. Utilizing loving touch, residents with dementia become cooperative with care and that makes staff work less challenging and increases their job satisfaction. 


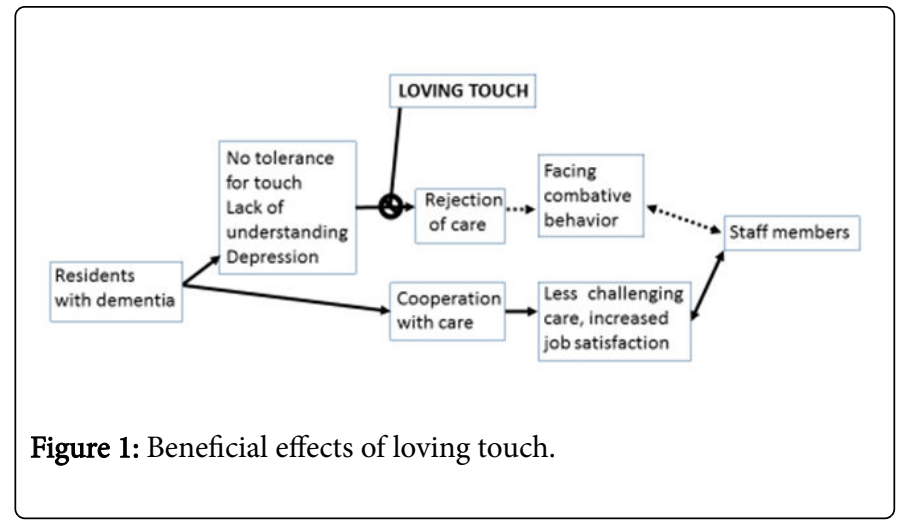

Touch is necessary for human development and children who are not touched die from failure to thrive [12]. Residents of a long-term care facility are often isolated, and if they do not have families that visit often, their main contact is with nursing assistants who are trained to complete tasks. These tasks often involve uncomfortable procedures and are performed in an institutional environment. Loving touch, provided outside of a task oriented care activity, may help residents to restore connection with other people and improve their quality of life. It is also an inexpensive treatment for rejection of care that does not have any adverse effects.

\section{References}

1. Ishii S, Streim JE, Saliba D (2012) A conceptual framework for rejection of care behaviors: review of literature and analysis of role of dementia severity. J Am Med Dir Assoc 13: 11-23.

2. Afram B, Stephan A2, Verbeek H3, Bleijlevens MH3, Suhonen R4, et al. (2014) Reasons for institutionalization of people with dementia: informal caregiver reports from 8 European countries. J Am Med Dir Assoc 15: 108-116.

3. Cohen-Mansfield J and Jensen B (2008) Physicians' perceptions of their role in treating dementia-related behavior problems in the nursing home: actual practice and the ideal. Journal of the American Medical Directors Association 9: 552-557.

4. Galindo-Garre F, Volicer L, Van der Steen JT (2015) Factors related to rejection of care and behaviors directed towards others: a longitudinal study in nursing home residents with dementia. Dement Geriatr Cogn Dis Extra 5: 123-134.

5. O'Connor DW, Ames D, Gardner B, King M (2009) Psychosocial treatments of psychological symptoms in dementia: a systematic review of reports meeting quality standards. Int Psychogeriatr 21: 241-251.

6. Morgan DG, Cammer A, Stewart NJ, Crossley M, D'Arcy C, et al. (2012) Nursing aide reports of combative behavior by residents with dementia: results from a detailed prospective incident diary. Journal of the American Medical Directors Association 13: 220-227.

7. Simard, J (2013) The End-of-Life Namaste Program for People with Dementia. (2nd)Health Professions Press,Baltimore, London, Sydney.

8. Manzar BA and Volicer L (2015) Effects of Namaste Care: Pilot Study. American Journal of Alzheimer's Disease 2: 24-37.

9. Stacpoole M, Hockley J, Thompsell A, Simard J, Volicer L (2015) The Namaste Care programme can reduce behavioural symptoms in care home residents with advanced dementia. Int J Geriatr Psychiatry 30: 702-709.

10. Fullarton J, Volicer L (2013) Reductions of antipsychotic and hypnotic medications in Namaste Care. J Am Med Dir Assoc 14: 708-709.

11. Soliman A and Hirst S. Using sensory activities to improve dementia care.

12. Polan HJ, Ward MJ (1994) Role of the mother's touch in failure to thrive: a preliminary investigation. J Am Acad Child Adolesc Psychiatry 33: 1098-1105. 